\title{
El método de presentación del estado de resultados utilizado por las sociedades anónimas chilenas y las motivaciones de su elección
}

\section{CECILIA DEL PILAR GALLEGOS-MUÑOZ ${ }^{a}$, ESTELA IRENE RODRÍGUEZ-QUEZADA ${ }^{b}$}

\begin{abstract}
RESUMEN La apertura de los mercados ha creado una nueva necesidad en materia contable: generar información comprensible y comparable a inversionistas o acreedores extranjeros. Esto implica un proceso de armonización de información financiera, el que en Chile inicia con las entidades supervisadas por la Superintendencia de Valores y Seguros, las cuales, de manera gradual a partir del año 2009, debieron adoptar las Normas Internacionales de Información Financiera (NIIF). Entre estas normas, cobra vital importancia la Norma Internacional de Contabilidad n. ${ }^{\circ} 1$ (NIC n. ${ }^{\circ}$ 1) (International Accounting Standards Board, IASB, 2006). En este contexto, el presente trabajo muestra los resultados de una investigación cuyo objetivo fue identificar la alternativa de presentación de estados de resultados utilizada por las sociedades anónimas chilenas, y cuáles fueron las motivaciones para su elección. Para el análisis de la información se utilizaron técnicas cuantitativas y cualitativas. La principal conclusión es que la mayoría de las sociedades anónimas abiertas en Chile presentan sus estados de resultados por función de los gastos, sin que exista una relación directa entre la alternativa escogida y el sector industrial al que esta pertenece. Las motivaciones para esta elección son: el ahorro en capacitación para empleados, la generación de información útil para la toma de decisiones y, mayoritariamente, la similitud de esta alternativa con el formato ya conocido como normativa contable chilena.
\end{abstract}

\section{HISTORIA DEL ARTÍCULO}

¿CÓMO CITAR?:

TGallegos-Muñoz, C. P. \& RodríguezQuezada, E. I. (2017). El método de presentación del estado de resultados utilizado por las sociedades anónimas chilenas y las motivaciones de su elección. Perspectiva Empresarial, 4(1), 73-84. http://dx.doi.org/10.16967/rpe. v4n1a5

RECIBIDO: 2 de marzo de 2016 APROBADO: 30 de enro de 2017

CORRESPONDENCIA:

Estela Irene Rodríguez-Quezada, Avenida Andrés Bello No 720 , Facultad de Ciencias Empresariales, Universidad del Bío-Bío, Campus Fernando May, Ciudad Chillán, Chile.

PALABRAS CLAVE estado de resultados, función de los gastos, naturaleza de los gastos, NIC n. ${ }^{\circ} 1$, sociedades anónimas abiertas.

a Magíster, profesora en la Universidad del Bío-Bío, Chile. Correo electrónico: cecilia@ubiobio.cl

b Magíster, profesora en la Universidad del Bío-Bío, Chile. Correo electrónico: erodrig@ubiobio.cl 
¿CÓMO CITO EL ARTÍCULO? HOW TO CITE THIS PAPER?

\section{CHICAGO:}

Gallegos-Muñoz, Cecilia del Pilar y Rodríguez-Quezada, Estela Irene. 2017. "El método de presentación del estado de resultados utilizado por las sociedades anónimas chilenas y las motivaciones de su elección". Perspectiva Empresarial 4(1): 73-84. http:// dx.doi.org/10.16967/rpe. v4n1a5

MLA:

Gallegos-Muñoz, Cecilia del Pilar y Rodríguez-Quezada, Estela Irene. "El método de presentación del estado de resultados utilizado por las sociedades anónimas chilenas y las motivaciones de su elección". Perspectiva Empresarial 4.1 (2017): 7384. Digital. http://dx.doi. org/10.16967/rpe.v4n1a5

\section{The Method Used by Chilean Limited Liability Companies to Present their P\&L Statement and the Reasons Behind It}

ABSTRACT Markets opening has created a new need in terms of accounting: to generate understandable and comparable data to investors or foreign creditors. The foregoing implies a process of harmonization in terms of financial information, which in Chile is conducted by the Superintendence of Securities and Insurance, which, gradually, as of 2009, started to adopt the International Financial Reporting Standards (IFRS). Among those standards, the International Accounting Standard N. ${ }^{\circ} 1$ (IAS n. ${ }^{\circ}$ ) (International Accounting Standards Board, IASB, 2006) is one of the most important. In such context, this paper shows the results of a research whose purpose was to identify the alternative used by Chilean companies at the moment of presenting their results, and the reasons behind it. To analyze the information, quantitative and qualitative techniques were used. The main conclusion is that most of the Chilean limited companies present their P\&L statements based on the expenses, without a direct relation between the chosen option and the industrial sector they belong to. The main reasons behind this situation have to do with saving money when training employees, the generation of useful information to make decisions and, especially, the similitude of this alternative with the format already known as the Chilean accounting standard.

KEYWORDS Profit and loss statement (P\&L), expense function, expense nature, IAS n. ${ }^{\circ}$, open limited companies.

\section{O método de apresentação do estado de resultados utilizado pelas sociedades anônimas chilenas e as motivações da sua eleição}

RESUMO A abertura dos mercados criou uma nova necessidade em matéria contável: gerar informação compreensível e comparável a investidores ou credores estrangeiros. Isto implica um processo de compatibilidade da informação financeira, que inicia no Chile com as entidades supervisionadas pela Superintendência de Valores e Seguros, as quais, de forma gradual a partir do ano de 2009, aprovaram as Normas Internacionais de Informação Financeira (NIIF). Entre estas normas, uma das mais importantes é a Norma Internacional de Contabilidade $\mathrm{n}^{0} 1\left(\mathrm{NIC}^{\circ}{ }^{\circ}\right.$ ) (International Accounting Standards Board, IASB, 2006). Neste contexto, o presente trabalho mostra os resultados de uma pesquisa com o objetivo de identificar a alternativa de apresentação de estados de resultados. Para a análise da informação se utilizaram técnicas quantitativas e qualitativas. A conclusão principal é que a maior parte das sociedades anônimas abertas no Chile apresentam os resultados segundo a função de gastos sem a relação direta entre a alternativa escolhida e o setor industrial ao que pertence. As motivações para esta escolha são: a economia na capacitação para empregados, a geração de informação útil para a tomada de decisões e, o mais importante, a semelhança desta alternativa com a já conhecida normativa contável chilena.

PALAVRAS CHAVE Mapa das despesas, função das despesas, natureza das despesas, "NIC no. 1", sociedades anônimas abertas. 


\section{Introducción}

En Chile, la Superintendencia de Valores y Seguros (SVS), como entidad fiscalizadora de las actividades que realizan las compañías que participan de los mercados de valores y seguros, publicó con fecha 27 de agosto de 2007 el calendario de adopción de las NIIF para las entidades fiscalizadas. En dicho calendario se señalaba que las sociedades anónimas abiertas debían acogerse de forma gradual a dichas normas a partir del $1^{\circ}$ de enero de 2009 , concluyendo el $1^{\circ}$ de enero de 2012 con la totalidad de las entidades fiscalizadas; estas debían presentar de forma obligatoria sus estados financieros según esta normativa. Cabe señalar que uno de los estados financieros que resulta más útil para la toma de decisiones gerenciales y que influye directamente en la decisión de los inversores es el estado de resultados, dado que proporciona un mayor nivel de detalle de los rendimientos obtenidos por la compañía.

Ahora bien, considerando la relevancia y el tipo de información que se puede obtener desde este informe, cabe tener presentes los dos métodos de presentación que propone la Norma Internacional de Contabilidad $n .{ }^{\circ} 1$ (en adelante NIC n. ${ }^{\circ} 1$ ): naturaleza de los gastos y función de los gastos. Cada uno tiene características particulares en cuanto a su esquema y a la manera en que la información financiera es presentada. La entidad debe optar por uno u otro método, decisión que se debe basar en una serie de criterios que pueden afectar la elección individual de cada organización. Sin embargo, de acuerdo con la literatura, el método escogido por la compañía pudiera influir en la decisión de los inversores, dado que el nivel de detalle difiere en cada uno de ellos.

Resulta entonces interesante identificar el método de presentación del estado de resultados que han utilizado las sociedades anónimas abiertas en Chile, después de cuatro años de haber sido exigida la aplicación de estas normas contables, y cuáles son los criterios que han considerado para su elección. El objetivo de esta investigación de carácter exploratorio es determinar el método de presentación de los estados de resultados utilizado por las sociedades anónimas abiertas en Chile, con el fin de establecer la relación existente entre el sector industrial al que pertenecen y el método de presentación empleado por las mismas, además de identificar los criterios que se tuvieron en cuenta para su elección.

\section{Revisión de la literatura}

Como todos los países, antes de la aparición de las NIIF, Chile aplicaba sus propias normas contables y de preparación de estados financieros, las cuales tenían como fuente dos instituciones: el Colegio de Contadores de Chile A.G. y la Superintendencia de Valores y Seguros (SVS). El Boletín Técnico . $^{\circ} 1$ del Colegio de Contadores de Chile (1973) establece el concepto "estados financieros", señalando que son "el medio por el cual la información cuantitativa acumulada, procesada y analizada por la contabilidad es periódicamente comunicada a aquellos que la usan", e indica que los estados financieros básicos son el balance general o estado de situación financiera y el estado o cuenta de resultado (Colegio Contadores de Chile A.G., 1973, p. 4). Por su parte, la Circular n. ${ }^{\circ}$ 1.501, publicada por la SVS en el año 2000, contenía las normas sobre forma y contenido de los estados financieros de las sociedades inscritas en el registro de valores, e indicaba que estos debían prepararse de acuerdo con los principios contables generalmente aceptados que emite el Colegio de Contadores de Chile A.G., siempre que dichos principios no contradigan los emitidos por la propia SVS - caso en el cual priman los últimos sobre los primeros-, y establecía un formato para presentar los estados financieros llamado ficha estadística codificada uniforme (FECU), que debía contener la identificación de la sociedad, el balance general, el estado de resultados, el estado de flujo de efectivo y las notas explicativas a los estados financieros (Superintendencia de Valores y Seguros, 2000, p. 1).

García (2003, p.158) señala que, en la actualidad, todos los países tienen como exigencia legal la elaboración y presentación de la información relativa a la situación de las empresas, y que en cada país existe una normativa que ha sido planteada de acuerdo con las características propias de cada nación, con el objetivo de regular el contenido y la forma de presentación de los estados financieros; estos son el medio de comunicación entre la empresa y los usuarios de la información. Dicha autora indica que esto permite que internamente la información contable sea comprensible y comparable, pero que surge un problema cuando esa información va dirigida a inversionistas o acreedores extranjeros, que solo conocen la normativa de su país, la cual puede diferir de la vigente en el país de origen de la información, lo que implica rehacer los estados financieros para 
poder adaptarlos a la normativa vigente del país destinatario. Así mismo, García (2003) señala que "estos problemas, que afectan a la totalidad de las empresas multinacionales, han llevado a la necesidad y factibilidad de contar con normas contables internacionales que permitan la armonización contable y guíen la preparación y presentación de esa información".

El surgimiento de estos problemas es la base primordial para la creación de una norma común, que establezca la forma en que las empresas deben presentar sus estados financieros. Amat \& Perramon (2005, p. 10) señalan que "la problemática de las diferencias contables entre países produce incredulidad en los usuarios de las cuentas", por lo que plantean que las normas internacionales vienen a mejorar la calidad de la información contable, estableciendo criterios que se deben cumplir para hacer frente a los problemas de contabilidad actual, tales como el deber de generar información que sea comparable a nivel internacional, y el de entregar información que sea fiable reduciendo las posibilidades de que se lleve a cabo la "contabilidad creativa" o los "maquillajes" (supuestamente legales).

En este proceso de armonización desempeña un papel importante el Comité de Normas Internacionales de Contabilidad (International Accounting Standards Committee, IASC), que tiene su origen en 1973, y cuyo objetivo es la formulación de una serie de normas contables que pudieran ser aceptadas y aplicadas con generalidad en distintos países, con la finalidad de favorecer la armonización de los datos y su comparabilidad. Este organismo trabajó hasta el 2000 en esa misión y, para sustituirlo, en abril del 2001 fue creada la Junta de Normas Internacionales de Contabilidad (International Accounting Standards Board, IASB). Amat \& Perramon (2005, p. 9), definen la IASB como un organismo de carácter privado, cuyo principal objetivo es la emisión de normas contables que puedan utilizarse en todo el mundo con el fin de llegar a tener un lenguaje contable único a nivel global.

El marco conceptual emitido por la IASB el año 2010 abarcó, entre otros aspectos, el objetivo de los estados financieros, donde se señala que este es "suministrar información acerca de la situación financiera, desempeño y cambios en la posición financiera. Se pretende que tal información sea útil a una amplia gama de usuarios al tomar sus decisiones económicas" (IASB, 2010, p. 5). Esta información se puede obtener desde el balance general o estado de situación financiera y el estado de resultados. En este escenario, en Chile se decide adoptar estas Normas Internacionales de Información Financiera como una forma de homogeneizar la información presentada por las empresas, comenzando con las sociedades anónimas abiertas, entidades chilenas supervisadas por la Superintendencia de Valores y Seguros. Estas sociedades debían presentar, a partir del año 2009, sus estados financieros de forma comparativa bajo la modalidad NIIF plenas o completas (Full IFRS). Ante esta situación, la SVS impartió nuevas instrucciones acerca del formato de presentación de los estados financieros que debían emitir las entidades fiscalizadas por ella. Con fecha 28 de diciembre de 2007, emitió el Oficio Circular n. ${ }^{\circ} 427$, que indicaba que a las entidades que debieran consolidar sus estados financieros con los de sus filiales solo se les exigiría presentarlos de manera consolidada, no siendo obligatoria la presentación de estados financieros individuales, y modificó la Circular n. $^{\circ} 1.501$ emitida en el año 2000, a través de la Circular n. 1.879 del 25 de abril del 2008. Finalmente adecuó el antiguo formato de presentación FECU a las nuevas normas, denominándolo FECU-IFRS (SVS, 2007, p. 3).

La Norma Internacional de Contabilidad referente a la presentación de los estados financieros (NIC n. ${ }^{\circ} 1$ ), tiene como objetivo recomendar la base de presentación de dichos estados, de tal forma que se genere información comparable entre estados financieros de la misma entidad correspondientes a periodos anteriores, y también comparables con estados de otras entidades. Esta norma es aplicable a todas las compañías, ya sea que presenten estados financieros consolidados o estados financieros separados o individuales. Esta norma contiene instrucciones acerca de la estructura que deben tener los estados financieros, y uno de ellos es el estado de resultados.

Al respecto, Guzmán, Guzmán \& Romero (2005, p. 164) postulan que el estado de resultados "pretende ofrecer, a través de un informe, la posibilidad de evaluar la rentabilidad que obtuvo un negocio durante un periodo determinado". También señalan que: "El estado de resultados debe ser presentado con un grado de detalle importante, para obtener así información útil para el análisis financiero de la empresa". En tanto, Ávila (2007, p. 68) indica que la finalidad del estado de resultados es la de "dar a conocer los resultados que en determinado periodo obtiene la negociación o empresa en todas sus operaciones 
de compra y venta de mercancías, mismas que a veces serán utilidades y a veces pérdidas". Al respecto, Horngren, Sundem \& Elliot (2000, p. 143) plantean que "a los inversionistas en general les interesa la capacidad de la compañía para producir utilidades y dividendos durante mucho más tiempo, información que es posible recabar del estado de resultados", por lo que se le considera un documento mucho más importante que el balance general.

La NIC $n .{ }^{\circ} 1$, en su párrafo $n .^{\circ} 99$, indica que los costos y gastos de una entidad se clasificarán para su presentación en el estado de resultados, de acuerdo con la naturaleza de los mismos o según la función que cumplan dentro de la entidad, estableciendo para ello dos métodos de presentación: "Método de la naturaleza de los gastos" y "Método de la función de los gastos". En el primer método, los gastos se agruparán en la cuenta de resultados de acuerdo con su naturaleza (esencia específica, por ejemplo, amortización, compras de materiales, costes de transporte, retribuciones a los empleados y costes de publicidad) y no se redistribuirán atendiendo a las diferentes funciones que se desarrollan en el seno de la entidad. Otal \& Serrano (2006, p. 335) mencionan que: "Este método resulta simple de aplicar, puesto que no es necesario proceder a distribuir los gastos de explotación entre las diferentes funciones llevadas a cabo dentro de la empresa". En cambio, en el segundo método, los gastos se presentan según la función que cumplan dentro de la entidad, como por ejemplo: costo de ventas, gastos de ventas y gastos de administración. En esta clasificación se revela el costo de ventas de forma separada de los otros gastos. Además, se establece que, al utilizar este método, de igual forma se debe revelar información adicional de la naturaleza de los gastos, incluidos los gastos por depreciación y amortización y el gasto por beneficios a los empleados, debido a que la naturaleza de los gastos es útil a la hora de predecir los flujos de efectivo.

Otal \& Serrano (2006, p. 327) hacen énfasis en una consideración especial que se debe tener al decidir utilizar el método de la función de los gastos, indicando que, en ocasiones, si bien este método "puede suministrar a los usuarios información más relevante que la ofrecida por la naturaleza de los gastos (...)", hay que tener en cuenta que "la distribución de los gastos por función puede resultar arbitraria, e implicar la realización de juicios subjetivos".
La NIC n. 1 indica que la entidad desglosará sus gastos para presentarlos en el estado de resultados, eligiendo el método que le "proporcione información más fiable y relevante". También señala que la elección entre el método por función o por naturaleza de los gastos depende tanto de factores históricos como del sector industrial donde se enmarque la entidad. Además, establece que "ambos métodos proporcionan una indicación de los costos que puedan variar directa o indirectamente con el nivel de ventas o de producción de la entidad", señalando que ambos presentan información útil para las sociedades que los utilizan, dependiendo generalmente del sector industrial al que pertenezcan (IASC, 2006, p. 17).

Al respecto, Otal \& Serrano (2006, p. 335) manifiestan que la descomposición de los gastos por función presenta ciertas ventajas: permite evaluar la evolución de los gastos por departamentos, evita perder la visión analítica derivada de la evolución de los gastos por naturaleza y aporta información acerca de los costos.

Al revisar la literatura desde el enfoque de la adopción de las normas NIIF (IFRS en inglés) y la aplicación de la NIC n. ${ }^{\circ} 1$ en la presentación de los estados de resultados, es posible notar la escasez de estudios que entreguen una visión de cómo ha sido tratado este tema, cuál es la tendencia que sigue con respecto al método de presentación elegido, cuál es el nivel de conocimiento, análisis o interpretación que le dan las empresas a esta situación y, finalmente, la complejidad de definir el avance, si es que lo ha habido, de cómo las entidades han cumplido con la norma en lo que respecta a la presentación del estado de resultados y desde el prisma de las ventajas y desventajas de prepararlo con uno de los dos métodos señalados en la NIC $n .^{\circ} 1$.

Sin perjuicio de lo anterior, hay estudios de los cuales emanan ciertas directrices que pueden ayudar a definir el aporte de la presente investigación al área temática que se está tratando. Así, se puede citar, en primer lugar, el estudio realizado por Yáñez, Pilar-Cortés, Inostrosa-Gonzales (2010, p. 56), en el cual se procede al análisis de los efectos que produciría la implementación de las NIIF con una muestra de 16 empresas chilenas no financieras cotizadas en la Bolsa de Santiago en el año 2008 y que adoptaron la normativa internacional en 2009. En dicho estudio fue posible observar que el $50 \%$ de las empresas presentó el estado de resultados por el método función de los gastos, mientras que la otra mitad lo hizo por el 
método naturaleza de los gastos. Según Kvaal \& Nobes (2010), la implementación de IFRS en un país no garantiza uniformidad de criterios, ya que existen diferencias sistemáticas en las políticas contables en cada uno de ellos.

En materia de métodos de presentación de estados financieros de acuerdo con las normas internacionales, existen algunos aportes en la literatura a nivel internacional, como son los de Ding, Jeanjean \& Stolowy (2008), quienes realizaron un estudio a 199 empresas que cotizan en Francia, con el objetivo de determinar las motivaciones que tienen estas compañías en la elección del método de presentación de sus estados financieros, llegando a la conclusión que la principal motivación es la internacionalización, influenciada por varios factores como el tamaño, la posibilidad de ser auditada internacionalmente y la normativa contable existente, entre otras. Por su parte, Duque, Muñoz \& Osorio (2011, p. 24) hacen un análisis separado de cada uno de los métodos establecidos por las NIIF, señalando que el principal error del método de la naturaleza de los gastos es pensar que los conceptos que se consumen en el área productiva son similares a los consumidos en las áreas de administración, de ventas o de logística. Añaden que otro error en el que incurren las empresas es "considerar que por ejemplo todos los beneficios que se pagan a los empleados tienen la misma naturaleza, y todos se pueden agrupar para efectos de la presentación del estado de resultados", estableciendo que, a su juicio, se trata de un error basándose en la teoría general de costos, porque según esta, "aquellos conceptos que se consumen en el área operativa tienen la 'naturaleza' de estar asociados al producto, mientras que los que se consumen en las áreas administrativas y de ventas tienen la 'naturaleza' de estar asociados al periodo". Además, establecen que "el objetivo de este método es ofrecer información más detallada, pero se queda corto en sus aspiraciones". La razón para llegar a esta conclusión radica en que "es muy difícil de utilizar en empresas que manejan inventarios (manufactureras) por lo que su uso se limitaría a las empresas comerciales o de servicios". Añaden que, en conclusión, "este método en lugar de agrupar los conceptos de acuerdo con su naturaleza, lo que hace es agrupar conceptos que por naturaleza deberían estar separados". En cuanto al método de la función de gastos, las conclusiones aportadas se resumen en que "frente a la información adicional que debe acompañar esta modalidad de estado de resultados, la NIC n. ${ }^{\circ}$
1 no fue específica ya que señala que al menos se debe mostrar el importe por gastos de depreciación y amortización, y el gasto por beneficios a empleados". El artículo de Duque, Muñoz \& Osorio concluye que la norma internacional intentó incluir el estado de costos en el estado de resultados presentado, pero este objetivo no se logró, e hizo que la utilidad de este informe interno se desvirtuara al presentar los estados de resultados con el método de naturaleza de los gastos.

Finalmente, Maines \& McDaniel (2000) analizaron cómo el formato de presentación del estado de resultados influye en la decisión de los inversores, y llegaron a la conclusión de que la decisión de estos se encuentra basada en patrones psicológicos, y se requiere un mayor nivel de detalle de información para tomar la decisión, dado que no se cuenta con los conocimientos técnicos suficientes para el análisis de los estados presentados.

\section{Metodología}

La presente investigación se realizó tanto desde el paradigma de investigación cuantitativo como cualitativo, utilizando técnicas de revisión documental, análisis gráfico y encuestas. Para determinar la población se utilizaron las 196 sociedades anónimas que publicaron sus estados financieros, tanto consolidados como individuales, en el sitio de la Superintendencia Intendencia de Valores y Seguros al 31 de diciembre de 2012, utilizando formato ficha estadística codificada uniforme (FECU-IFRS).

Respecto al análisis cuantitativo, se dividió la población de 196 sociedades según la actividad económica desarrollada, en cuatro grupos: empresas comerciales, industriales, servicios y otros. En esta última clasificación se incluyeron aquellas que presentaron sus estados financieros consolidados, y que cuentan con filiales que desarrollan diversas actividades económicas. Con estas sociedades se utilizó como mecanismo la revisión documental de los estados financieros presentados por dichas sociedades anónimas y el análisis de las notas explicativas presentes en la Memoria anual, que hacen públicas a través de la página web de la Superintendencia de Valores y Seguros, procedimiento para analizar la forma en que estas sociedades presentaron sus estados de resultados y el sector industrial al cual pertenecían estableciendo relaciones entre ambas variables. 
Ahora bien, con el fin de darle un carácter explicativo a esta investigación, se procedió a llevar a cabo un análisis más profundo de estas sociedades, aplicando una encuesta cuyo objetivo fue determinar las motivaciones de la elección de dicho método de presentación. Para lo anterior, se procedió a enviar una invitación a participar de la investigación a las 196 sociedades, recibiendo respuestas positivas de diez de ellas, que manifestaron su interés en participar de la investigación. Estas diez sociedades constituyen el objeto de estudio, y a estas se procedió a aplicar una encuesta a las personas responsables de la confección de los estados financieros en estas entidades, que permitiera conocer las motivaciones que tuvieron para la elección del método de presentación de estado de resultados por utilizar en dicha empresa.

\section{Resultados}

De la población total de sociedades anónimas que presentaron sus estados financieros con formato FECU-IFRS al 31 de diciembre de 2012, 155 empresas los emitieron de manera consolidada y 41 lo hicieron en forma individual, lo que corresponde a $79 \%$ y $21 \%$, respectivamente. En cuanto a la fecha de la primera emisión de estados financieros según la Norma Internacional, del mismo total de 196 sociedades se obtuvo la siguiente información: El 65\% de ellas (128 sociedades) presentó sus estados financieros bajo la NIIF por primera vez en el año 2009. El 25\% de ellas (49 sociedades) lo hizo en 2010, y el 8\% (15 sociedades) en 2011. El 2\% restante (cuatro sociedades) los presentó por primera vez en el año 2012. Con respecto a la modalidad de presentación de los estados financieros que utilizaron la primera vez que los emitieron según la NIIF, se estableció que un 64\% de la población (126 sociedades) emitió sus estados financieros con la modalidad Full IFRS, contra el 36\% restante, que lo hizo según la modalidad pro forma.

En cuanto al método de presentación del estado de resultados utilizado, se observa que un $15 \%$ lo hizo por el método de la naturaleza de los gastos, lo que corresponde a 29 sociedades, y las 167 restantes lo hicieron por el método de función de los gastos, lo que representa un $85 \%$ del total. Ahora bien, de las 29 sociedades que presentaron sus estados de resultados al 31 de diciembre de 2012 por el método de la naturaleza de los gastos se encuentran todas las Administradoras de Fondos de Pensiones privadas existentes hoy en Chile (cinco AFP), cinco sociedades del rubro telecomunicaciones, seis empresas del rubro electricidad y tres empresas relacionadas con el procesamiento y distribución de agua potable. Las diez sociedades restantes son de diversos rubros, entre ellas tres agroindustrias, dos sociedades de inversión y otros. Así mismo, se pueden mencionar dos situaciones que advierten una relación directa entre empresas y su método de presentación de estados de resultados. Tal es el caso de la Sociedad de Inversiones Tricahue S.A., cuyo objeto social exclusivo es "el de adquirir y vender acciones de la Empresa Eléctrica Pehuenche S.A. o de quien legalmente la suceda". En dicho caso, ambas empresas presentan su estado financiero de manera individual utilizando el método de la naturaleza de los gastos para la elaboración de su estado de resultados. El otro caso por señalar es el de la Sociedad Almendral S.A., en la cual "su principal activo corresponde a su participación en Entel Chile S.A., equivalente a más del $98 \%$ de sus inversiones". En este caso ambas empresas, Entel Chile S.A. y Almendral S.A., presentan sus estados financieros de manera consolidada al 31 de diciembre de 2012, y ambas exponen su estado de resultados por el método de la naturaleza de los gastos. Al realizar este mismo análisis según el tipo de estado financiero presentado, ya sea individual o consolidado, se observa que el método más utilizado sigue siendo el método de la función de los gastos, el que en el caso de los estados financieros individuales representa un 83\%, con un total de 34 sociedades que utilizaron este método, en tanto que en los estados financieros consolidados representa un $86 \%$, con un total de 133 sociedades. Al separar la población total según la actividad económica desarrollada por la sociedad, se obtiene que son: 12 comerciales, 76 de servicios, 81 industriales y 27 clasificadas en otros, lo que representa $6 \%, 39 \%, 41 \%$ y $14 \%$, respectivamente. El método de presentación de sus estados de resultados más utilizado por cada grupo sigue siendo el de función de los gastos; sin embargo, se observa claramente en el sector de las empresas de servicios un aumento en la presentación de su estado de resultados por el método naturaleza de los gastos, donde un $25 \%$ correspondiente a 19 de estas empresas expuso su estado de resultados por este método, en tanto, en el resto de los grupos, solo una de las empresas comerciales, seis de las industriales y tres del grupo otros lo hicieron por este método, lo que representa un $8 \%, 7 \%$ y 
$11 \%$, respectivamente, del total de empresas de estos grupos.

Ahora bien, al analizar los resultados según el tipo de estado financiero presentado, ya sea individual o consolidado, se observa que la tendencia se mantiene en cuanto a que el método más utilizado por las empresas es el método función de los gastos, sin importar el tipo de actividad económica desarrollada. Se destacan las sociedades de servicios que presentaron estados financieros consolidados, donde 14 de ellas (27\%) expusieron su estado de resultados por el método naturaleza de los gastos, seguidas por las empresas del mismo sector pero que presentaron estados financieros individuales, con cinco sociedades $(21 \%$ del total) que emplearon este mismo método. Por otra parte, las empresas industriales y comerciales que presentaron sus estados financieros consolidados fueron las que menos utilizaron este método, con un total de cinco sociedades, lo cual equivale al $7 \%$ de las empresas industriales y a una sociedad $(8 \%)$ en el caso de las comerciales. Del mismo modo, se puede agregar que en las empresas clasificadas en el rubro otros, un 10\% (tres sociedades) presentan sus estados financieros mediante el método naturaleza de los gastos y el $90 \%$ restante lo sigue haciendo por el método función de los gastos.

En cuanto a los resultados de la encuesta aplicada a las sociedades sujeto de estudios, en primer lugar es importante señalar que esta fue respondida por funcionarios que desempeñaban cargos directamente relacionados con el área contable de la sociedad, dentro de los cuales se encontraban: contadores generales, analistas, asistentes de contabilidad y jefes del departamento de contabilidad, todos participantes en la preparación de los estados financieros. En lo relativo a la antigüedad, en los cargos de todos los responsables de la elaboración de los estados financieros en las sociedades es superior a cinco años en la empresa, lo cual permite aseverar que todos ellos, dados los años de antigüedad y las funciones desempeñadas, tienen pleno conocimiento respecto a las preguntas que contestaron.

Por otra parte, un elemento que vale la pena considerar en la preparación de los informes financieros que las sociedades anónimas abiertas deben presentar a la SVS es el software utilizado. Respecto a esto, y antes de la adopción de la norma internacional, las sociedades anónimas estudiadas en profundidad señalaron en su mayoría que antes de la aplicación de IFRS utilizaban un software comprado para preparar sus informes financieros. Sin embargo, luego de la adopción de IFRS, la mayoría de estas empresas debió adaptar el software contable usado para la emisión de sus informes o adquirir un nuevo software para poder preparar y elaborar los estados financieros con esta nueva normativa.

En relación con el conocimiento de los métodos de presentación de estados de resultados que señala la NIC $n .{ }^{\circ} 1$, todas las sociedades analizadas señalaron tener conocimiento de cuáles son dichos métodos. Sobre el método de presentación escogido para exponer el estado de resultados, señalan en su gran mayoría hacerlo con el método de la función de los gastos. En lo concerniente a las motivaciones que tuvieron para esta elección, indicaron que porque les permite ahorrar en capacitación a sus empleados, generar información de mejor calidad para la toma de decisiones y, mayoritariamente, porque este método presenta mayor similitud con el ya utilizado antes en la normativa chilena. Cabe destacar también que todas las sociedades, independientemente del método de presentación escogido para presentar el estado de resultados, cumplen con la obligación de revelar en las notas explicativas a los estados financieros la naturaleza de dichos gastos, mostrados en el estado de resultados. Ahora bien, en cuanto a la utilidad que se le da a la información que se obtiene de dicho método de presentación, los encuestados señalaron que lo aplicaban:

i) como apoyo y respaldo de la contabilidad de gestión tanto de la sociedad como del gobierno corporativo;

ii) para análisis del directorio;

iii) para dar cumplimiento a la normativa, $\mathrm{y}$

iv) para la gestión financiera.

En tanto, las empresas que manifestaron escoger como método de presentación de su estado de resultados el método de la naturaleza de los gastos, expresaron que esta elección se debe a que ese método genera información de mejor calidad para la toma de decisiones, y que la utilidad que se le da a la información proporcionada por este método es que sirve de apoyo contable y para la toma de decisiones gerenciales.

Cabe destacar que ninguna de las sociedades estudiadas en profundidad indicó presentar, explícitamente, su estado de resultado bajo ambos métodos. 
Por último, en cuanto a la opinión de los encuestados acerca de las ventajas y desventajas de la implementación de las NIIF, estos señalan que los beneficios se resumen en que:

i) se produjo un mayor nivel de análisis y desarrollo de las partidas del balance y estado de resultado;

ii) se obtienen los valores reales en cuanto a contabilizar a valor justo, $y$

iii) existe mayor claridad y comparabilidad de la información financiera.

Respecto de las desventajas, se destacan las siguientes:

i) mayores costos en: compra/adaptación de software para reportar a SVS (XBRL), consumo de tiempo en la confección de los estados financieros, e incremento en gastos por concepto de honorarios para auditoría y capacitación, y

ii) la no aplicación de la corrección monetaria, lo que conlleva una mayor diferencia en la determinación de la renta líquida imponible para efectos tributarios.

Finalmente, todos los encuestados concuerdan en que la implementación de las NIIF han clarificado la interpretación de la información financiera proporcionada por las sociedades anónimas.

\section{Conclusiones}

Según los resultados obtenidos, se observa que existe una fuerte inclinación de las sociedades anónimas abiertas por presentar sus estados financieros mediante el método función de los gastos. Algunos autores señalan que utilizar el método de la naturaleza no es recomendable para las empresas manufactureras o de servicios que manejan inventarios, pues es muy compleja su construcción, y no es útil a la hora de tomar decisiones, por lo que solo sería aplicable en empresas comercializadoras. Para el caso de Chile, se observó que del total de la población, seis empresas cuya actividad económica es la manufactura presentaron su estado de resultados por naturaleza de los gastos, entre ellas tres del sector eléctrico (generación) y tres del sector agroindustrial. Esta situación llama especialmente la atención puesto que estas últimas son empresas que aun manteniendo niveles de inventarios y pudiendo haber utilizado el método por función (el cual se utilizaba tradicionalmente en Chile antes de la aplicación de las NIIF), optaron por un mayor nivel de revelación utilizando el método por naturaleza de los gastos, lo que a llevó a las autoras de este artículo a preguntarse cuáles serían las motivaciones de las sociedades a la hora de escoger un método de presentación de estado de resultados.

Al observar los resultados del análisis explicativo, se comprobó que la principal motivación para la elección del método de presentación de estado de resultados por función de los gastos tiene que ver con el hecho de que los encargados de la preparación y presentación de los estados financieros en las sociedades anónimas consideran que este método es similar al que utilizaban antes de la adopción de las normas IFRS, lo que facilita también la adaptación con los softwares contables existentes en la compañía. Así mismo, se puede establecer, a raíz de los resultados del análisis cualitativo, que los criterios que utilizan las sociedades anónimas abiertas a la hora de seleccionar el método de presentación de su estado de resultados, son en orden de prioridad: optimización de tiempo en la preparación de dicho estado financiero, ahorro en costos de adaptación del software contable utilizado y generación de información, entendiéndose por esta la que sea útil para la toma de decisiones. Esto explicaría por qué las empresas estudiadas emplean mayoritariamente el método de función de los gastos.

La literatura señala que el método por naturaleza debiera, dadas las características de la información financiera que esta maneja, ser utilizado principalmente por empresas de servicios. En este sentido, se observó que son estas las que mayoritariamente avanzan en la presentación de su estado de resultados por este método, ya que de todas las empresas que lo hicieron con el método naturaleza de los costos, 19 de un total de 29 (66\%), pertenecían al sector económico de servicios, destacándose principalmente las de servicios sanitarios, empresas distribuidoras de electricidad y Administradoras de Fondos de Pensiones (AFP). En este último caso, las cinco AFP que existen en Chile lo presentan usando el método naturaleza de los gastos.

A partir de las investigaciones y análisis expuestos anteriormente, aun cuando el método más utilizado resultó siempre el de función de los gastos, se pudo comprobar que la decisión de su elección se toma principalmente considerando 
las posibilidades de generación de información con las que cuentan en la actualidad las sociedades anónimas debido a los sistemas informáticos que estas utilizan, y los cuales no se encuentran preparados según los parámetros que las NIIF requieren; además, su adaptación implicaría una alta inversión para las entidades informantes, y no porque este método les proporcione información más fiable y relevante como señala la literatura, o porque sea más útil para la decisión de los inversionistas, o por el sector industrial al que pertenece, y muchas veces se realiza sin un estudio previo de las partidas que se pueden ver afectadas.

También se demostró que, en opinión de los encuestados, la aplicación de las normas internacionales, si bien es cierto que ayudó a clarificar la interpretación de la información financiera, para algunas entidades ha significado mayores costos que beneficios, como son costos en compra y adaptación de softwares para reportar a la SVS, consumo de tiempo en la confección de los estados financieros, incrementos en gastos de honorarios para auditoría y capacitación, entre otros.

Finalmente, se evidencia que existen tres empresas clasificadas dentro del sector otros que presentan sus estados de resultados usando el método naturaleza de los gastos. Ahora bien, si se analizan las características de las empresas que fueron clasificadas en este rubro, se trata principalmente de sociedades de inversión y holding con rubros diversificados, por lo cual surgió en las autoras de este documento la siguiente interrogante para este tipo de empresas en las que sus filiales desarrollan actividades económicas de diversa categoría: ¿Cuál sería el método más apropiado considerando que deben consolidar sus estados financieros y mantener las mismas políticas contables entre matriz y filiales consolidadas?

\section{REFERENCIAS}

Amat, O. \& Perramon, J. (2005). Normas Internacionales de Contabilidad: NIC/NIIF (1a. ed.). España: Gestión 2000.

Ávila, J. (2007). Introducción a la contabilidad. México: Umbral.

Bolsa de Comercio de Santiago (2013). Consulta emisores de valores. Recuperado de http://www. bolsantiago.cl

Colegio de Contadores de Chile A.G. (1973). Boletín Técnico $n .^{\circ} 1$. Santiago, Chile.
Ding, Y., Jeanjean, T., \& Stolowy, H. (2008). The impact of firms' internationalization on financial statement presentation: Some French evidence. Advances in Accounting, incorporating. Advances in International Accounting, 24, 145-156.

Duque-Roldán, M., Muñoz-Osorio, L. \& Osorio-Agudelo, J. (2011). El estado del costo de producción y venta y el estado de resultados en las Normas Internacionales de Información Financiera NIIF/IFRS. Universidad de Antioquia.

García, M. T. (2003). Fundamentos y normativa de la contabilidad. Santiago: Lexis Nexis.

Guzmán, A., Guzmán, D., \& Romero, T. (2005). Contabilidad financiera. Colombia: Centro Editorial Universidad del Rosario.

Horngren, Ch., Sundem, G. \& Elliot, J. (2000). Introducción a la contabilidad financiera (7ä. ed.). México: Pearson Educación de México, S.A. de C.V.

International Accounting Standards Board, IASB. (2006). Presentación de estados financieros. Norma Internacional de Contabilidad $n .^{\circ} 1$ (NIC $n .{ }^{\circ} 1$ ).

International Accounting Standards Board, IASB. (2010). Marco conceptual para la información financiera.

Kvaal, E., \& Nobes, C. (2010). International differences in IFRS policy choice: a research note. Accounting and Business Research, 40(2), 173-187.

Maines, L. A., \& McDaniel, L. S. (2000). Effects of Comprehensive-Income Characteristics on Nonprofessional Investors' Judgments: the Role of Financial-Statement Presentation Format. The Accounting Review, 75(2), 179-207.

Ministerio de Educación (1958). Ley n..$^{\circ}$ 13.011: Crea una institución con personalidad jurídica, denominada Colegio de Contadores, Chile.

Ministerio de Hacienda (1981). Decreto Ley n..$^{\circ} 3538$ : Crea Superintendencia de Valores y Seguros. Última versión 01.02.2012. Santiago, Chile.

Morales, F. \& Jarne, J. (2006). Los avances de la armonización contable internacional en Argentina, Brasil y Chile. Revista Contabilidad y Auditoría, 173.

Otal, S. \& Serrano, R. (2006). Las Normas Internacionales de Información Financiera: Análisis y aplicación. Capítulo 3: Presentación de estados financieros. España: Aranzadi S.A.

Superintendencia de Valores y Seguros (2000). Circu$\operatorname{lar} .^{\circ} 1.501$.

Superintendencia de Valores y Seguros (2007). Oficio Circular n. 427.

Superintendencia de Valores y Seguros (2008). Circular n. ${ }^{\circ} 1.879$.

Superintendencia de Valores y Seguros (2013). Estados financieros de 196 sociedades anónimas abiertas. Recuperado de http://www.svs.cl/portal/principal/605/w3-channel.html 
Superintendencia de Valores y Seguros (2013). Calendario de adopción de Normas IFRS en Chile. Recuperado de http://www.svs.cl/sitio/legislacion_normativa/normativa/doc/calendario_adopcion_ifrs.pdf
Yáñez-Andrade, V., Pilar-Cortes, F. \& Inostrosa-Gonzales, C. (2010). Impacto de las IFRS en la valorización de empresas: evidencia para una muestra de empresas chilenas cotizadas. Revista Activos. 
